\title{
Gastrointestinal Symptoms of COVID-19
}

\author{
Sun Jiawei* \\ Shulan International Medical College, Zhejiang Shuren University, China
}

*Corresponding author: Sun Jiawei, Shulan International Medical College, Zhejiang Shuren University, Hangzhou, Zhejiang, China. To Cite This Article: Sun Jiawei, Gastrointestinal Symptoms of COVID-19. 2020 - 11(1). AJBSR.MS.ID.001616.

DOI: 10.34297/AJBSR.2020.11.001616.

Received: 䟧 December 09, 2020; Published: 些 December 14, 2020

\begin{abstract}
COVID-19 caused by SARS-COV-2, first reported in China in December 2019, has now affected the entire world. Some studies have found that gastrointestinal symptoms are common clinical symptoms in COVID-19 patients. To explore the correlation between gastrointestinal symptoms and symptoms, diagnosis, treatment, and prognosis of COVID-19 is of great significance.
\end{abstract}

Keywords: COVID-19, Digestive system

\section{Introduction}

Novel coronavirus (COVID-19 pneumonia) is causing a global pandemic, which is also one of the major challenges facing human health at present. It is caused by novel coronavirus (2019-nCoV) infection and belongs to $\beta$ - coronavirus (including SARS-CoV and Severe Respiratory Syndrome Coronavirus (MERS-CoV)). After infection, patients usually have fever and respiratory diseases, but some patients may have gastrointestinal symptoms such as diarrhoea, vomiting and abdominal pain.

\section{Digestive Symptoms in Patients with COVID-19}

At present, COVID-19 pneumonia is spread by respiratory droplets or contact, with respiratory symptoms as the first symptom while some with digestive tract symptoms as the first symptom [1]. Among 1602 cases of pneumonia in COVID-19, 55 cases (5.6\%) suffered diarrhoea, and 72 cases $(4.5 \%)$ suffered nausea or vomiting. SARS-CoV-2 enters cells via the angiotensin converting enzyme 2 (ACE2) receptor and is highly homologous to SARS-CoV [2]. The researchers found 2019-nCoVRNA in stool specimens of COVID-19patients, and its receptor angiotensin-converting enzyme 2 (ACE2) was highly expressed in gastrointestinal epithelial cells, suggesting that 2019-nCoV can be actively infected and replicated in the gastrointestinal tract. This finding has important implications for the management, transmission, and infection control of 2019nCoV [3]. At the beginning of 2020, a study published in BioRxiv analyzed 4 databases containing sequencing results of lung, esophagus, stomach, ileum, and colon transcriptome. The results showed that ACE 2 was highly expressed in intestinal epithelial cells of ileum and colon, lung cells and stratified epithelial cells of upper esophagus. In addition, compared with healthy adults, the expression of ACE 2 in intestinal epithelial cells of patients with colorectal adenoma or colorectal cancer was higher, suggesting that these patients are more susceptible to COVID-19 pneumonia [4]. A recently published single cell RNA sequencing study also found that ACE 2 is mainly over-expressed in bile duct cells, which may cause abnormal liver function. At the same time, another study said that nearly half of the pneumonia patients in COVID-19 suffered from liver damage (mainly ALT and AST increased), which may be caused by SARS-CoV-2 infection or antiviral drugs.

Although there is no conclusive evidence that SARS-COV-2 can be transmitted through feces, it has been successfully isolated from faeces and anal swab samples of infected patients [5].

\section{Consensus on Digestive Management During} COVID-19 Pneumonia

In fact, doctors involved in the diagnosis and treatment of pneumonia in COVID-19 at present have different experiences in diagnosis and treatment of digestive diseases and symptoms. According to a survey of 2209 digestive physicians in China, the 
correct rate of experts to COVID-19 knowledge about digestive system damage is about $31 \% \sim 35 \%$, which means that their knowledge and dynamic progress on respiratory system damage of pneumonia in COVID-19 are not enough [6]. In view of the need for clinical treatment, the increase of overseas morbidity and imported cases, and the potential crisis of local cases, it is necessary to reach a consensus on the diagnosis and treatment of pneumonia digestive system in COVID-19.

Recently, the consensus on digestive management during the epidemic period in novel coronavirus was published. It put forward some practical suggestions on digestive symptoms and management methods as well as digestive outpatient service and related process management in COVID-19, which deserves the attention of us [5].

The following points are mentioned in the consensus. Some patients with COVID-19 do have digestive tract symptoms, which are related to virus infection, inflammation, stress, and drug side effects. Fever control, drug management, liver support, psychotherapy and enteral nutrition can be used to manage upper gastrointestinal symptoms of patients. According to reports, the new diarrhoea after infection with 2019-nCoV (excluding diarrhoea caused by adverse drug reactions) can reach $22.2 \%$ [7] The use of montmorillonite and specific probiotics can improve the diarrhoea of patients. Antibiotic-related diarrhea and Clostridium diffile infection (CDI) should also be vigilant. Statistics show that the incidence of abnormal liver function in pneumonia in COVID-19 can reach $39.1 \%$ [7] and $43.4 \%$ [8], so we should pay attention to the dose of antiviral drugs in the management of liver injury. When most patients have slight abnormal liver function (no more than 2 times of normal value), they only need to dynamically observe the changes of liver function without medication; For liver injury caused by antiviral drugs, priority should be given to pneumonia. If liver function is abnormal, it should be treated with liver protection, such as reduced glutathione, glycyrrhizic acid derivatives and their compound preparations. About half of COVID-19 patients' appetite decreased obviously. Nutritional support therapy is one of the key measures in the absence of specific drugs for pathogens. Oral administration of enteral nutrition is recommended. Patients with digestive tract diseases can supplement parenteral nutrition and maintain normal energy supply to tolerate enteral nutrition.
The gastrointestinal fluids and feces of COVID-19 patients may be infectious, so they should be protected in the practice of digestive clinic, and the patients should be screened, and risk stratified preliminarily. At the same time, to prevent the spread of virus, it is suggested that selective endoscopy and faecal bacterial transplantation should be suspended or postponed in epidemic areas.

As 2019-nCoV has strong communicability, it poses a serious threat to global human health. In the early stage of the outbreak of the disease, medical staff focused on examining respiratory symptoms. However, with the understanding of the disease enhanced, digestive system symptoms related to COVID-19 pneumonia have been gradually confirmed. At present, it is not clear whether the virus in digestive system comes from cell fragments of respiratory system or is copied from digestive tract, so it is wise to prevent faecal-oral transmission in hospitals and communities.

\section{References}

1. Wang D, Hu B, Hu C, Zhu F, Liu X, et al. (2020) Clinical Characteristics of 138 Hospitalized Patients With 2019 Novel Coronavirus-Infected Pneumonia in Wuhan, China. JAMA 323(11): 1061-1069.

2. Wu F, Zhao S, Yu B, Yan Mei Chen, Wen Wang, et al. (2020) A new coronavirus associated with human respiratory disease in China. Nature 579(7798): 265-269.

3. Ma C, Cong Y, Zhang H (2020) COVID-19 and the Digestive System. Am J Gastroenterol 115(7): 1003-1006.

4. Lian Yong Li, Wei Wu, Sheng Chen, Jian Wen Gu, Xin Lou Li, et al. (2020) Digestive system involvement of novel coronavirus infection: Prevention and control infection from a gastroenterology perspective. Journal of Digestive Diseases 21(4): 199-204.

5. Zhang X, Tang C, Yang Y, Tian D, Hou X (2020) Management of Digestive Disorders and Procedures Associated With COVID-19. Am J Gastroenterol 115(8): 1153-1155.

6. Nunoo Mensah JW, Maeda K, Chun HK, Pasquale Giordano, Richard Fortunato (2020) COVID-19 and the Global Impact on Colorectal Practice and Surgery. Clin Colorectal Cancer 19(3): 178-190.

7. Sun J, Aghemo A, Forner A, Valenti L (2020) COVID-19 and liver disease. Liver Int 40(6): 1278-1281.

8. Chen N, Zhou M, Dong X, Jieming Qu, Fengyun Gong, et al. (2020) Epidemiological and clinical characteristics of 99 cases of 2019 novel coronavirus pneumonia in Wuhan, China: a descriptive study. Lancet 395(10223): 507-513. 